\title{
Efficient Extraction of Multiple Kuratowski Subdivisions
}

\author{
Markus Chimani, Petra Mutzel, and Jens M. Schmidt \\ Department of Computer Science, University of Dortmund, Germany \\ \{markus.chimani, petra.mutzel, jens.schmidt\}@uni-dortmund.de
}

\begin{abstract}
A graph is planar if and only if it does not contain a Kuratowski subdivision. Hence such a subdivision can be used as a witness for non-planarity. Modern planarity testing algorithms allow to extract a single such witness in linear time. We present the first linear time algorithm which is able to extract multiple Kuratowski subdivisions at once. This is of particular interest for, e.g., Branch-and-Cut algorithms which require multiple such subdivisions to generate cut constraints. The algorithm is not only described theoretically, but we also present an experimental study of its implementation.
\end{abstract}

\section{Introduction}

A planar drawing of a graph is an injection of its vertices onto points in the plane, and a mapping of the edges into open curves between their endpoints. These curves are not allowed to touch each other, except in their common endpoints. Graphs which admit such a planar drawing, are called planar graphs, and recognizing this graph class has been a vivid research topic for the past decades. Hopcroft and Tarjan [11] showed in 1974 that this problem can be solved in linear time, using sophisticated data structures and intricate algorithms. Current planarity testing algorithms like the ones by Boyer and Myrvold 445] and de Fraysseix et al. 910 are less complex but still quite involved.

As shown by Kuratowski 13] in 1930, a graph is planar if and only if it does not contain a $K_{3,3}$ or a $K_{5}$ subdivision, i.e., a complete bipartite graph $K_{3,3}$ or complete graph $K_{5}$ with edges replaced by paths of length at least one. Such subgraphs are called Kuratowski subdivisions. The efficient extraction of such a witness of non-planarity was non-trivial in the context of the first linear planarity tests. A linear algorithm for such an extraction was later presented, e.g., by Williamson [15]. Modern planarity testing algorithms like the ones by Boyer and Myrvold, and de Fraysseix et al. can directly extract a single Kuratowski subdivision, if the given graph is non-planar.

In ILP-based Branch-and-Cut approaches which try to solve, e.g., the Maximum Planar Subgraph problem [12] or the Crossing Minimization problem [6], the identification of multiple such witnesses is a crucial part. Thereby, we look at some intermediate solution and try to find Kuratowski subdivisions. For each such subdivision, we can try to generate a cut constraint, necessary to efficiently 
solve the ILP. Experience shows that it is desirable to find multiple Kuratowski constraints at once, as they strengthen the LP-relaxation of the problem.

In the following, let $G=(V, E)$ be a non-planar undirected graph, without selfloops and multi-edges. Current planarity tests are able to extract a single Kuratowski subdivision in linear time $O(n), n:=|V|$. We address the problem of finding multiple Kuratowski subdivisions in efficient time. As there may exist exponentially many Kuratowski subdivisions in general, it is not practical to enumerate all of them. A basic approach would be to obtain $k$ Kuratowski subdivisions through calling a planarity test $k$ times and subsequently deleting an involved Kuratowski edge. This approach has a superlinear runtime of $O(k n)$, but we are not aware of any algorithm faster than this approach, up until now.

In this paper, we propose an algorithm which extracts multiple Kuratowski subdivisions in optimal time $O\left(n+m+\sum_{K \in \mathcal{S}}|E(K)|\right)$, with $\mathcal{S}$ being the set of identified Kuratowski subdivisions and $m:=|E|$. This runtime is linear in the graph size and the extracted Kuratowski edges. The algorithm is based on the planarity test of Boyer and Myrvold [5] which is one of the fastest planarity tests today [3. We will only give a short introduction into this planarity test in Section 2, for a full description of the original test see [5]. The main part of this paper focuses on the description on how to modify and extend all steps to obtain multiple subdivisions in linear time, which requires both algorithmic changes, as well as a heavily modified runtime analysis. Finally, Section 4 gives a short computational study which shows the effectiveness of this algorithm.

\section{The Boyer-Myrvold Planarity Test}

The test starts with a depth first search on the (not necessarily connected) input graph, which divides the edge set into DFS-forest edges and into backedges, pointing to nodes with smaller depth first index DFI. The aim is to construct a planar drawing based on the DFS-forest, by successively embedding all backedges in descending DFI order of their end vertices. Throughout this paper, let $v$ be the current vertex to embed. Any backedge ending on $v$ is called pertinent and will be embedded, if this is possible while maintaining planarity. In the beginning, each DFS-edge is separated from its adjacent vertex with lower DFI and joined to a new virtual vertex. Therefore it represents a biconnected component (bicomp) in the beginning, which grows when backedges are embedded.

To identify involved bicomps during such an embedding, the Walkup is called for each start node of a pertinent backedge. A bicomp consisting of only one DFS-edge and its adjacent vertices is called degenerated. The Walkup marks the involved subgraph and classifies nodes as pertinent and external: a node $w$ is called pertinent, if there exists a pertinent backedge $\{w, v\}$ or if $w$ has a child bicomp in the DFS-tree which contains a pertinent node. A node $w$ is called external, if there exists a backedge $\{w, u\}$ with $u$ having a smaller DFI than $v$, or if $w$ has a child bicomp containing an external node. Bicomps are called pertinent or external if they contain pertinent or external vertices, respectively. The Walkup traverses a unique path from $w$ to $v$ on the external faces of bicomps 
for every pertinent backedge $\{w, v\}$. We denote this path as the backedge path of $\{w, v\}$.

The Walkdown attempts to embed each pertinent backedge and merges the bicomps between its start and end vertex in the DFS-tree to a new, larger bicomp. It is invoked twice for each child bicomp of $v$ : once in a counterclockwise direction around the external face of the child bicomp, and once in the clockwise direction. Using the classification of nodes from the Walkup, the Walkdown embeds only backedges which preserve planarity in the embedding. If any backedge cannot be embedded, the graph is not planar and a subdivision is extracted; otherwise a planar embedding is found. Since non-embeddable backedges can only occur when both Walkdowns stop on external vertices which are not pertinent, such a situation is called a stopping configuration. We call unembedded pertinent backedges caused by a stopping configuration critical. Let $b=\{w, v\}$ such a critical backedge. The first node in the backedge path of $b$ which is contained in the same bicomp as both stopping vertices are, is called critical node. We denote the part of the backedge path from $w$ to this critical node critical back path.

\section{Extracting Multiple Kuratowski Subdivisions in Linear Time}

As opposed to the Boyer-Myrvold planarity test, the number of edges cannot be bounded linearly by the number of vertices. Since every algorithm has to read the input graph and to output all identified Kuratowski subdivisions, $\Omega(n+m+$ $\left.\sum_{K \in \mathcal{S}}|E(K)|\right)$ is a lower bound for the runtime and our algorithm is therefore optimal for the extracted number of Kuratowski edges.

\subsection{Overview}

The original planarity test terminates when a stopping configuration is found. It is possible to extract a Kuratowski subdivision for each critical backedge of this stopping configuration. To obtain more, we have to proceed with the algorithm. This bears problems, since the embedding has to be maintained planar, which is impossible if it contains Kuratowski subdivisions. The idea is to identify all critical backedges in the given stopping configuration and delete them. After that, the bicomp $B$ containing the stopping configuration is not pertinent anymore and it is necessary to continue at the situation directly before the planarity test descended to $B$. This allows finding the next stopping configuration, provided that there exists any on the current embedding step of vertex $v$. See Algorithm 1 for an overview of these steps for the embedding of a single vertex $v$.

Unfortunately, almost all time-bounds given in [5] loose validity with this approach, and a new runtime analysis of this extended algorithm is necessary. The key to a linear time bound is to compensate additional costs during Walkup, Walkdown and extraction by the amount of extracted Kuratowski edges.

We will first describe how to find the correct reentry point after a stopping configuration was found and removed. In Section 3.3, we discuss how to modify 


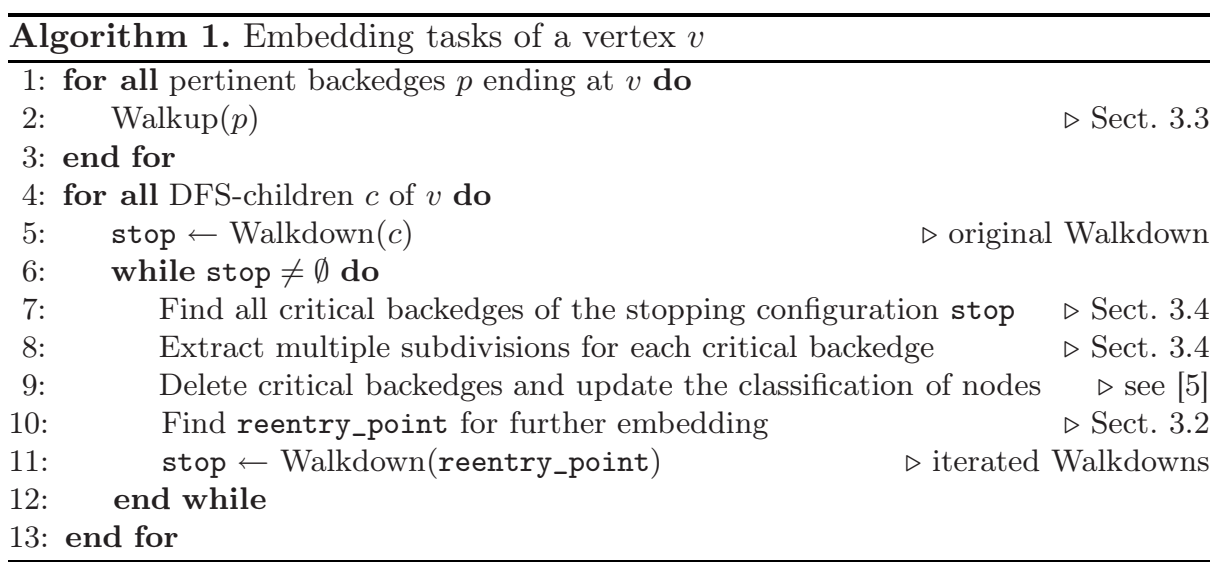

the Walkup, in order to allow efficient operations used in the later steps of the algorithm. Section 3.4 deals with the efficient extraction phase. Finally, the overall runtime of the extended algorithm is analyzed in Section 3.5.

Of course there are graphs with exactly one Kuratowski subdivision. Hence, we do not ensure any lower bound other than 1 for the number of extracted Kuratowski subdivisions of non-planar graphs. But in practice, the quantity is high as discussed in Section 4, Formally, our algorithm guarantees:

Lemma 1. We find at least one unique Kuratowski subdivision for each critical backedge per stopping configuration.

Lemma 2. Whenever the algorithm extracts a Kuratowski subdivision using a critical backedge $b$, and there exists at least one additional Kuratowski subdivision without b, we will find such a subdivision.

\subsection{Finding the Reentry Point for Further Embeddings}

Let $v^{\prime}$ be the virtual node of $v$ adjacent to the DFS-child $c$ of $v$ from the current Walkdown. We call the bicomp which has $v^{\prime}$ as its root, the forebear bicomp, the others are called non-forebear bicomps. The Walkdown can be run unmodified, as long as no stopping configuration occurs. The same holds if a stopping configuration occurs on the forebear bicomp due to embedded pertinent backedges, since this represents the last stopping configuration in the Walkdown.

Otherwise, the Walkdown has to be modified. Let $A$ be the non-forebear bicomp containing the stopping configuration, $T$ the subtree of all pertinent bicomps with the bicomp containing $v^{\prime}$ as root and $D$ the parent bicomp of $A$ in $T$ (cf. Figure 11). Any bicomp in $T$ has exactly those bicomps as children which are referenced in the PertinentRoots lists of its nodes, as proposed in [5]. In Figure 1] the bicomp tree $T$ consists of the (degenerated) forebear bicomp $\left\{v^{\prime}, c\right\}$ and the non-forebear bicomps $A, B, C$ and $D$. The Walkdown stops at $A$, deleting the critical backedges incident to $w_{1}$ and $w_{2}$ after the extraction of all Kuratowski subdivisions induced by these backedges. Afterwards, $A$ is not 
pertinent anymore and its PertinentRoots list entry on the parent node $z_{1}$ in $D$ must be deleted. As there exists another item in that list, we continue the Walkdown at $z_{1}$ and find another stopping configuration in bicomp $B$. The general rule is that the Walkdown continues on $z_{1}$ until the PertinentRoots list of $z_{1}$ is empty.

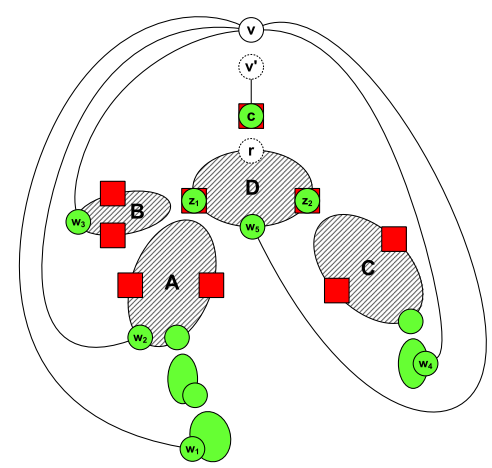

Fig. 1. Finding reentry points. Square nodes refer to external vertices; circular, light gray nodes denote pertinent vertices. Virtual vertices are depicted by a dotted line.

At last, $z_{1}$ is not pertinent anymore. Furthermore, short-circuit edges from the root $r$ of $D$ to both external vertices in each direction $\left(z_{1}\right.$ and $\left.z_{2}\right)$ have been embedded.These short-circuit edges permit an $O(1)$-traversal to the other external vertex $z_{2}$, where the Walkdown extracts all stopping configurations of child bicomps (bicomp $C$ in Figure 1), analogously to $z_{1}$. Finally, we check whether $D$ itself contains a stopping configuration by extracting all remaining critical edges. In our example, the backedge starting at $w_{5}$ induces a subdivision and can be deleted after the subdivision's extraction. This procedure is iterated for the next father bicomp in the DFS-tree until the forebear bicomp is reached or a pertinent backedge is embedded. In the latter case, all preceding bicomps are embedded and the Walkdown continues at the forebear bicomp.

The crucial point in this scheme is the traversal to a bicomp, where no backedge can be embedded, i.e., a bicomp that contains a stopping configuration: we modify the embedding to what it would have been, if no critical backedges on this bicomp would have existed. Finally, the Walkdown is restarted on the very node where the previous Walkdown started to descent to this bicomp.

\subsection{Walkup}

Additionally to the PertinentRoots list and BackedgeFlags of the original planarity test, we now have to collect some more information during the Walkup. For every visited node $n$, we store a link LinkToRoot to the root node of the bicomp of $n$. This can be done efficiently by using a stack for all visited nodes of the bicomp during the Walkup. Furthermore, a list named PertinentNodesAfterWalkup of all pertinent nodes of each bicomp $B$ is created. This is stored at the root node of $B$ by collecting the nodes during the Walkup in a list. Whenever we reach the bicomp root or a node with set LinkToRoot, we can add the collected vertices in $O(1)$ time to the list of the bicomp root. Once established, this list is not modified until $v$ is completely embedded.

It is useful to be able to distinguish the backedges incident to different virtual vertices $v^{\prime}$ of $v$, since they will be embedded in different subtrees later on. This can be done by storing $v^{\prime}$ as the HighestVirtualNode for each backedge $\{w, v\}$. 
To obtain $v^{\prime}$ for a given backedge $p$, Walkup $(p)$ marks each visited node with $p$. If the Walkup ends on a virtual node of $v$, we can store this node as the HighestVirtualNode $(p)$. Otherwise, $\operatorname{Walkup}(p)$ stopped on an already visited vertex which was traversed during the Walkup of another backedge $q$. Since both Walkups met, the subtrees are identical and so are the HighestVirtualNodes of $p$ and $q$. The latter can be looked up in $O(1)$, and we hence identified HighestVirtualNodes $(p)$. This allows us to easily generate a list BackedgesOnVirtualNode for each virtual node $v^{\prime}$ of $v$ containing the backedges belonging to the pertinent subtree with root $v^{\prime}$.

\subsection{Extraction}

Overview. The extraction starts whenever the Walkdown halts on some stopping configuration in a bicomp $B$. We describe how the critical backedges of this stopping configuration can be computed in the next subsection "Extraction of Critical Backedges". Each critical back path of those backedges induces one or more Kuratowski subdivisions of a specific minor-type, which has to be known prior to the extraction. To obtain this minor-type, a path from each stopping vertex to a node with lower DFI than $v$ is selected in time linearly to its length.

Additionally, the highest-xy-path of the critical node $w$ is needed to determine the minor-type. As defined by Boyer and Myrvold, the highest-xy-path obstructs the inner face of $B$ and consists of the external face part on the top of the former, now embedded, bicomp which contains $w$. This path can be computed in $O(n)$, but this would result in a superlinear overall runtime. Hence we develop a more efficient way by first extracting the more general highest-face-path efficiently and use it to obtain the highest-xy-paths for all critical nodes. These steps are described in the subsections "Extraction of the Highest-Face-Path" and "Extraction of all Highest-XY-Paths". After the minor-type is determined, all remaining parts of the Kuratowski subdivision can be extracted from the DFStree using only external faces of involved bicomps. This requires time linearly to their lengths. Finally, all critical backedges of the stopping configuration as well as the involved PertinentRoots and BackedgeFlags are deleted. We will give a rather high level description of the extraction, referring the reader to [7,14 for technical details and case distinctions.

Extraction of Critical Backedges. Let $x$ and $y$ be the two stopping vertices on the bicomp $B$, and $r$ the root of $B$. Neither $x$, nor $y$, nor any node on the external face paths $r \rightarrow x$ and $r \rightarrow y$ can be pertinent; otherwise the Walkdown would not have stopped at $x$ and $y$. The critical back paths of the critical backedges end on the external face of $B$ between $x$ and $y$. We distinguish between two cases depending on the type of $B$.

If $B$ is a forebear bicomp, all pertinent backedges of the current Walkdown are contained in the BackedgesOnVirtualNode $(r)$ list. For each entry, we can check in $O(1)$ whether it is embedded. If not, the backedge is critical. This yields an overall running time of $O(n+m)$ over all embedding steps, since all critical backedges are deleted afterwards and no further stopping configuration can exist. 
If $B$ is a non-forebear bicomp, consider the DFS-subtree $T$ of pertinent bicomps with $B$ as root bicomp. We start a preorder traversal through $T$ by using the PertinentNodesAfterWalkup lists on the roots of all bicomps. These lists can contain nodes that are not pertinent any more due to extractions of other stopping configurations. Hence we have to check each item for pertinence; every non-pertinent entry is deleted. The remaining nodes are the critical nodes and we check their BackedgeFlag property. If this flag is set, the associated backedge must be critical and is therefore included in the list of critical backedges. Note that the remaining nodes, independent of their BackedgeFlag, may have nonempty PertinentRoots lists. After all critical backedges starting at the current bicomp were found, the preorder traversal iterates the process on each child bicomp given by its PertinentRoots lists recursively.

All tests on the nodes can be performed in constant time. The size of the tree $T$ itself is bounded by the costs of the corresponding Walkup invocations, because at least one node was traversed for each pertinent bicomp. Moreover, a nonpertinent node in the PertinentNodesAfterWalkup list can only happen as a result of an earlier extracted stopping configuration. The only other reason would be that a pertinent backedge has been embedded on $B$, which contradicts the assumption. Each of the at most $m$ stopping configurations in all embedding steps produces at most one non-pertinent entry in a PertinentNodesAfterWalkup list. Hence the overall runtime is bounded by the Walkup time.

Independent of the case distinction on $B$, all critical nodes in $B$ are necessary for the minor-type classification and for the extraction of Kuratowski subdivisions. We can obtain all critical nodes in $B$ efficiently by testing the BackedgeFlag for each entry of the PertinentNodesAfterWalkup list of $r$. From the above description we can conclude:

Lemma 3. The asymptotic runtime for obtaining all critical backedges of a stopping configuration is bounded by the Walkup costs.

Extraction of the Highest-Face-Path. In order to extract all highest-xypaths efficiently, we first require a highest-face-path of the bicomp B. See Figure2 for a visualization of the following explanations. We obtain the highest-face-path by temporarily deleting all edges incident to its root $r$ except for the two edges $s=(r, a)$ and $t=(r, b)$ on the external face (ignoring any short-circuit edges). Thereby, $B$ breaks into multiple sub-bicomps; we also delete all separated subbicomps, i.e., the sub-bicomps which do not contain $r$. Consider the inner face $f$ containing $a, r$, and $b$. The highest-face-path is the path $a \rightarrow b$ on the boundary of $f$ not traversing $r$.

It is possible to extract the highest-face-path in time $O(|B|)$, if $B$ is properly embedded. But since the planarity test performs implicit flips on bicomps, we do not know whether the adjacency lists of the nodes are in clockwise or counterclockwise order, and we would have to establish the correct orientation for each node of $B$ first. This requires a traversal of the underlying DFS-tree, resulting in a superlinear overall runtime. Hence, this approach is not suitable and we will identify the highest-face-path with inconsistent node orientations instead. 


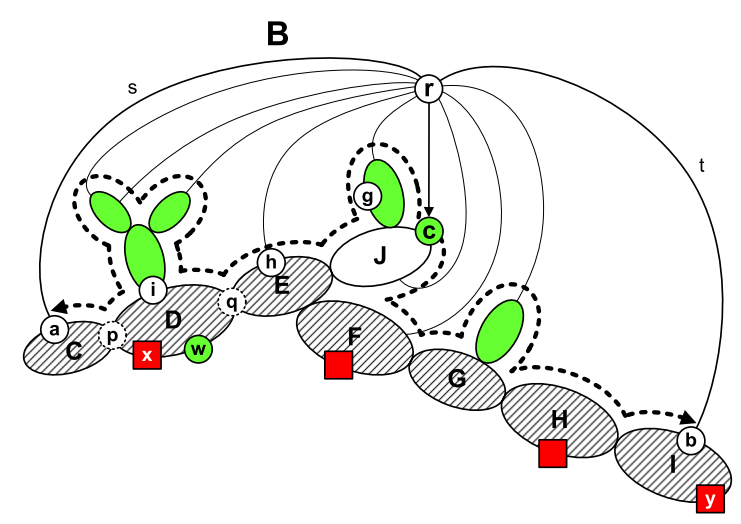

Fig. 2. The structure of the bicomp $B$ containing former bicomps. The hatched former bicomps form the bottom chain. The extraction of the highest-face-path starts at the inner vertex $c$ in both directions (thick dotted arrow lines) and ends on nodes $a$ and $b$.

Therefore, it is not possible to easily walk along $f$. The idea is to reuse the external face links, which were introduced in the original planarity test, of the former, now merged bicomps in $B$. These external-links of a node referred to the two incident edges on the boundary and could be used in a traversal of the external face in order to find the correct direction to proceed, even when some nodes are not oriented correctly. Unfortunately, the Walkdown will usually modify those external-links. Therefore, we store a backup copy old-links of the external-links on each bicomp root during the Walkup.

To use the former external-links in a traversal inside of a non-degenerated $B$, we have to analyze the general structure of $B$ first: the external face of every non-degenerated forebear bicomp contains at most one embedded backedge for each of the two Walkdowns formerly started at $r$. It may also contain an edge connecting the root and the non-root node with least DFI. However, in all cases these edges are incident to the virtual root node. The remaining set of edges on the external face consists of the lower parts of now connected, former bicomps. We denote this sequence of former bicomps which lie on the external face the bottom chain of $B$, cf. Figure 2, A merge node is a node shared between two adjacent bicomps of the bottom chain (e.g. the nodes $q$ in Figure 21), or one of the two end nodes $a$ and $b$. Given a former bicomp $U$ in the bottom chain, the path on the upper part of $U$ connecting the two contained merge nodes resembles the highest-xy-path of a critical back path ending at $U$. This fact is the key for the later extraction of all highest-xy-paths.

Let $c$ be the unique non-virtual node of $B$ with smallest DFI. Let $E$ be the former bicomp of the bottom chain which contains the node with smallest DFI: if $c$ is not contained in $E$, inner bicomps exist. Hence, we can summarize the necessary traversal as follows: We start with the traversal at $c$. If neither $s$ nor $t$ is an external-link of $c, c$ is either an inner vertex or the root of $E$ which lies on the external face of $B$. The former induces inner bicomps along a path from $c$ to 
the root of $E$. In both cases, we traverse the boundary of former bicomps in both directions. If an external-link of $c$ is either $s$ or $t, c$ lies on the external face, and we have to traverse only one direction, following the other external-link of $c$. If we use two traversal directions, $E$ can be determined as the last bicomp, whose root node is visited by both traversals. Starting with this root, all traversed nodes are stored in two separate lists, one for each traversal direction. We obtain the highest-face-path of $B$ by appending the reversed second list to the first one. All walks check on each visited node $z$ whether $z$ is identical to $a$ or $b$ in $O(1)$. If so, the walk is finished. During the traversal, all visited nodes are saved on a stack. If a node is visited twice, this node is a merge node to an inner, separated subbicomp, whose boundary is not part of the highest-face-path. Then, all nodes between the two occurrences are deleted from the stack.

We store the highest-face-path on the unique vertex $c$ in $B$, since later extractions might need it as well. Whenever a highest-face-path has to be computed in consequence of an embedding of $B$ within a larger bicomp $B^{*}, B$ will play the role of a former bicomp. Since we only traverse the external faces of former bicomps, we will never again traverse the interior of $B$. Hence, and since the traversals require $O(1)$ time for each vertex, we obtain:

Lemma 4. All highest-face-paths which occur during the algorithm can be computed and maintained in $O(n+m)$.

Extraction of all Highest-XY-Paths. For every given critical node $w$ between two stopping vertices of a stopping configuration, we have to compute its highest-xy-path. Let $D$ be the former bicomp of the bottom chain of $B$. By traversing the external face of $D$ from $w$ in parallel, using again the old-links, we find the merge nodes and extract the highest-xy-path in linear time of its length. For details see [7].

Extraction of Kuratowski Subdivisions. The prior sections dealt with the problem of efficiently obtaining and classifying multiple stopping configurations. We now address the problem to extract multiple Kuratowski subdivisions out of a single stopping configuration. Whenever a stopping configuration occurs, an appropriate critical back path for each critical backedge is computed. Along with the highest-xy-path, the minor-type of the induced Kuratowski subdivision is obtained. Additionally to the basic 9 minor-types of [5], we can define 7 more minor-types, by augmenting the types $B, C, D$ and $E_{1}-E_{4}$ with a non-empty path $v \rightarrow r$ as in type $A$. We call the resulting minor-types $A B, A C, A D$ and $A E_{1}-A E_{4}$, respectively. It turns out that the Kuratowski subdivisions of these additional minor-types constitute the largest part of the extracted subdivisions in practice, see Section 4. Clearly, more than one minor-type can exist for a single critical back path.

To further increase the number of extracted subdivisions, we will start with focussing on the critical back paths, since nearly all minor-types need them for constructing the subdivision. In general, such a path consists of external face parts between the roots of multiple consecutive bicomps. We can therefore extract the other parts of these external faces and combine these to obtain 
potentially exponentially many different critical back paths, which yield different Kuratowski subdivisions. As a side effect, those subdivisions are all similar which can be beneficial for the application area of Branch-and-Cut algorithms. The same technique can be used to obtain multiple external backedge paths and multiple paths starting at the so-called external z-nodes [5] in the minor-types $E_{1}-E_{5}$ and $A E_{1}-A E_{4}$.

All extracted Kuratowski subdivisions of a stopping configuration are unique. This holds for subdivisions of different stopping configurations as well, except for the minor-types $E_{2}$ and $A E_{2}$, which do not include the critical back path and thus might be extracted as minor-type $A$ later on. This can be avoided by a special marker on the external backedges, to prohibit its classification as a future critical backedge in $A$.

Bundle Variant. Moreover, we can extend our algorithm by a bundle variant in which all root-to-root paths of each involved bicomp on a critical back path are extracted. This approach increases the number of identified subdivisions dramatically, albeit on the cost of the running time. To speed up the backtracking subroutine, it is possible to use algorithms for dynamic connectivity for planar graphs [8]. This increases the overall runtime only by a factor of $\log (n)$ in comparison to the linear time approach in terms of output complexity.

\subsection{Runtime Analysis}

All steps described so far guaranteed an overall linear runtime. It remains to show that the modified Walkup can be bound by a linear total of $O(n+m+$ $\left.\sum_{K \in \mathcal{S}}|E(K)|\right)$. We will only give a brief sketch of the proof, and omit a number of rather technical case differentiations (see [7[14]).

It is sufficient to consider the costs of the Walkup, which cannot be compensated by new embedded faces or new short-circuit edges. Therefore, we only consider Walkup costs on critical backedge paths. If these are part of stopping configurations on non-forebear bicomps, the sum of all critical backedge-path costs on all forebear bicomps can be estimated as follows: we spend at most $O\left(n+m+\sum_{K \in \mathcal{S}}|E(K)|\right)$ time on the external face, and at most $O(m)$ time on inner faces containing the forebear root. Moreover, all other costs caused by stopping configurations in non-forebear bicomps are compensated by the inevitably induced minor $A$ which contains all other traversed edges.

Otherwise, the stopping configuration is contained in a forebear bicomp $B$. Since most minor-types do not contain the whole external face of $B$ in their Kuratowski subdivisions, all not yet compensated costs arise on its external face. The only exception to this rule are the critical paths on minors $E_{2}, A E_{2}$, which can be bound by a linear total as well. These remaining costs are compensated by the extracted Kuratowski paths of the different minor-types. Hence we yield Theorem 1, which is optimal in terms of output complexity. Based on this, we can furthermore deduce a corresponding result for the bundle variant.

Theorem 1. The overall running time of the algorithm is bounded by $O(n+$ $\left.m+\sum_{K \in \mathcal{S}}|E(K)|\right)$ and therefore linear. 
Corollary 1. The overall running time of the bundle variant is $O(n+m+$ $\left.\log n \sum_{K \in \mathcal{S}}|E(K)|\right)$.

\section{Experimental Analysis}

We implemented the algorithm and its bundle variant as part of the open-source $\mathrm{C}++$-based Open Graph Drawing Framework [1]. All tests were performed on an Intel Core2Duo with 1.86 GHz and 2GB RAM using the GNU-compiler gcc-3.4.4 (-o1). Due to the algorithmic complexities, we simplified the steps to compute the critical backedges and highest-xy-paths by correctly orienting $B$ in time $O(|B|)$. Although this simplification breaks the provable linear runtime, our experiments show that it does not influence the running time negatively in practice, since the number of extracted Kuratowski edges becomes the dominant term. The bundle variant uses a traditional back-tracking scheme and therefore does not guarantee the theoretical logarithmic bound. We use the Rome Graph Library [2, which contains 11528 real-world graphs with 10 to 100 nodes, 8249 of which are nonplanar graphs. We also use random graphs $(n=10 \ldots 500, m=2 n)$ generated by OGDF. Thereby we start with an empty graph on $n$ vertices and iteratively add an edge with random start and end node, until $m$ unique edges are added.

Each Rome graph is processed in less than $11 \mathrm{~ms}$ (on average: $1.3 \mathrm{~ms}$ ). The average amount of extracted Kuratowski subdivisions per 100-node graph is 255, containing in total 12214 Kuratowski edges. It is interesting that the average size of the subdivisions grows approximately with $n / 2$ throughout all tests. More Kuratowski subdivisions are obtained by the bundle variant. Thereby, each graph is processed in less than $1 \mathrm{sec}$ (but on average less than $7 \mathrm{~ms}$ ), extracting up to 3.5 million Kuratowski edges at some graphs (see Figure 3). There are 2912 subdivisions on average per 100-node graph with 136027 Kuratowski edges.

On the random graphs, the number of identified Kuratowski subdivisions increases dramatically for the bundle variant, such that a full computation becomes prohibitive. In practice, one can of course stop the computation after a certain amount of extracted subdivisions. Hence, we restrict our test to the linear variant for these random graphs (see Figure 3). Each graph needs less than $430 \mathrm{~ms}$

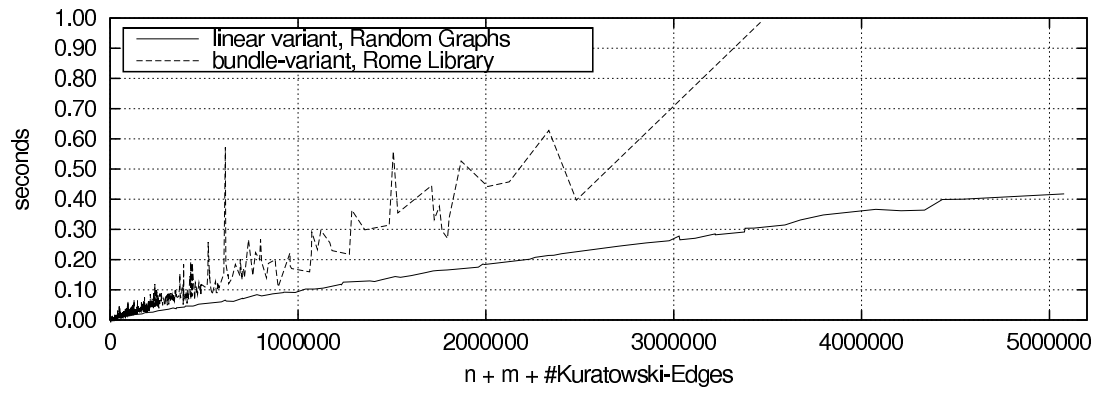

Fig. 3. Running times. The linear variant for the Rome Library would be nearly invisible in the very left corner of the figure. 
(126 ms on average), extracting up to 25000 Kuratowski subdivisions per graph containing 5 million Kuratowski edges. The average number of Kuratowski subdivisions is 8813 per graph with 1.3 million Kuratowski edges.

Overall, the experiments show a linear running time, despite the aforementioned simplifications of the algorithm. The minor-types are dominated by the types $A E_{1}-A E_{4}$, which constitute $60 \%-90 \%$ of all subdivisions on graphs with at least 100 nodes.

\section{References}

1. OGDF - Open Graph Drawing Framework. University of Dortmund, Chair of Algorithm Engineering, Website under Construction (2007)

2. Di Battista, G., Garg, A., Liotta, G., Tamassia, R., Tassinari, E., Vargiu, F.: An experimental comparison of four graph drawing algorithms. Comput. Geom. Theory Appl. 7(5-6), 303-325 (1997)

3. Boyer, J.M., Cortese, P.F., Patrignani, M., Di Battista, G.: Stop minding your P's and Q's: Implementing a fast and simple DFS-based planarity testing and embedding algorithm. In: Liotta, G. (ed.) GD 2003. LNCS, vol. 2912, pp. 25-36. Springer, Heidelberg (2004)

4. Boyer, J.M., Myrvold, W.J.: Stop minding your P's and Q's: A simplified O(n) planar embedding algorithm. In: Proc. SODA 1999, pp. 140-146. SIAM, Philadelphia, PA (1999)

5. Boyer, J.M., Myrvold, W.J.: On the cutting edge: Simplified O(n) planarity by edge addition. Journal of Graph Algorithms an Applications 8(3), 241-273 (2004)

6. Buchheim, C., Chimani, M., Ebner, D., Gutwenger, C., Jünger, M., Klau, G.W., Mutzel, P., Weiskircher, R.: A branch-and-cut approach to the crossing number problem. In: Healy, P., Nikolov, N.S. (eds.) GD 2005. LNCS, vol. 3843, pp. 37-48. Springer, Heidelberg (2006)

7. Chimani, M., Mutzel, P., Schmidt, J.M.: Efficient extraction of multiple Kuratowski subdivisions (TR). Technical Report TR07-1-002, Chair for Algorithm Engineering, Dep. of CS, University Dortmund (2007), http://ls11-www.cs.uni-dortmund.de/people/chimani/

8. Eppstein, D., Italiano, G.F., Tamassia, R., Tarjan, R.E., Westbrook, J.R., Yung, M.: Maintenance of a minimum spanning forest in a dynamic planar graph. J. Algorithms 13(1), 33-54 (1992)

9. de Fraysseix, H., de Mendez, P.O.: On cotree-critical and DFS cotree-critical graphs. Journal of Graph Algorithms an Applications 7(4), 411-427 (2003)

10. de Fraysseix, H., Rosenstiehl, P.: A characterization of planar graphs by Trémaux orders. Combinatorica 5(2), 127-135 (1985)

11. Hopcroft, J., Tarjan, R.: Efficient planarity testing. J. ACM 21(4), 549-568 (1974)

12. Jünger, M., Mutzel, P.: Maximum planar subgraphs and nice embeddings: Practical layout tools. Algorithmica 16(1), 33-59 (1996)

13. Kuratowski, K.: Sur le problème des corbes gauches en topologie. Fundamenta Mathematicæ 15, 271-283 (1930)

14. Schmidt, J.M.: Effiziente Extraktion von Kuratowski-Teilgraphen. Diploma thesis, Department of Computer Science, University of Dortmund (March 2007)

15. Williamson, S.G.: Depth-first search and Kuratowski subgraphs. J. ACM 31(4), 681-693 (1984) 